\title{
GUIA DE INSTALAÇÃO
}
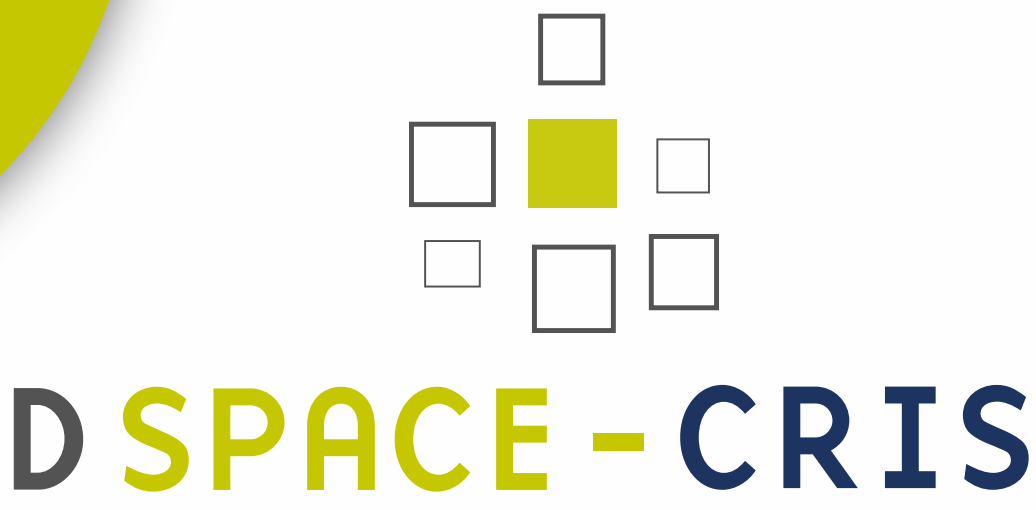

Lucas Silveira Milton Shintaku Andrea Bollini 
Guia de instalação DSpace-CRIS 
Instituto Brasileiro de Informação em Ciência e Tecnologia (Ibict)

Diretoria

Cecília Leite Oliveira

Coordenação-Geral de Pesquisa e Desenvolvimento de Novos Produtos (CGPD) Arthur Fernando Costa

Coordenação- Geral de Pesquisa e Manutenção de Produtos Consolidados (CGPM)

Lillian Alvares

Coordenação-Geral de Tecnologias de Informação e Informática (CGTI) Leonardo Lazarte

Coordenação de Ensino e Pesquisa, Ciência e Tecnologia da Informação (Coep) Lena Vania Ribeiro Pinheiro

Coordenação de Editoração (Coed)

Ramón Martins Sodomada Fonseca

Coordenação de Desenvolvimento de Sistema (Code)

Marcos Pereira Novais

Coordenação de Articulação, Geração e Aplicação de Tecnologia (Coat) Milton Shintaku 


\section{Lucas Ângelo da Silveira Milton Shintaku \\ Andrea Bollini}

\section{Guia de Instalação DSpace-CRIS}

Ibict

Brasília

2016 
(C) 2016 Ibict

Esta obra é licenciada sob Atribuição CC BY 4.0, sendo permitida a reprodução parcial ou total desde que mencionada a fonte.

\section{Editor Executivo}

Ramón Martins Sodoma da Fonseca

Assistentes de Editoração

Davilene Ramos Chaves

Gislaine Russo de Moraes Brito

\section{Revisão de Conteúdo}

Milton Shintaku

\section{Revisão Gramatical}

Margaret de Palermo Silva

Design Gráfico, Diagramação e llustrações

Andréa Maria de Castro Santos

Fleury Curado

\section{Normalização, Catalogação e}

Classificação

Ingrid Schiessl

\footnotetext{
Dados Internacionais de Catalogação-na-Publicação (CIP) S587

Silveira, Lucas Angelo da.

Guia de instalação DSpace-CRIS / Lucas Ângelo da Silveira; Milton Shintaku; Andrea Bollini . - Brasília: Ibict, 2016.

50 p.: il. color.

ISBN: 978-85-7013-118-8

http://dx.doi.org/10.18225/978-85-7013-118-8

1. Informática. 2. DSPACE-CRIS. 3. Software livre. 4. Instalação e configuração. I. Shintaku, Milton. II. Bollini, Andrea. III. Título.
}

CDU: 004.01(083.131)

Instituto Brasileiro de Informação em Ciência e Tecnologia (Ibict)

Setor de Autarquias Sul (SAUS)

Quadra 05 Lote 06, Bloco H-5oAndar

Cep:70.070-912 - Brasília,DF

Telefones:55 (61) 3217-6360/55 /

(61)3217-6350 www.ibict.br

Rua Lauro Muller, 455 - 4ㅇAndar - Botafogo CEP: 22.290 -160 - Rio de Janeiro,RJ 
Telefone:55(21)2275-0321

Fax:55(21)2275-3590

http://www.ibict.br/capacitacao-e-

ensino/pos-graduacao-em-ciencia-da-

informacao

http://www.ppgci.ufrj.br 


\section{Sumário}

2.1 CRIAR UM USUÁRIO

2.2 INSTALAR O BANCO DE DADOS

2.3 CRIAR A BASE DE DADOS

2.4 INSTALAR A JAVA VIRTUAL MACHINE (JVM)

3.1 CRIAR O DiRetório base

3.2 CRIAR O DIRETÓRIO FONTE

3.3 CONFIGURAR O DSPACE-CRIS PARA INSTALAÇÃO

3.4 COMPILAR O DSPACECRIS-FONTE

3.5 INSTALAR O IMAGEMAGICK 
8.1 ADICIONAR O IDIOMA PORTUGUÊS DO BRASIL

8.2 BUSCA INTERNA NOS ARQUIVOS ARMAZENADOS

8.3 Configurar SERVIÇO dE E-MAILS

8.3.2 DEFINIR PARÂMETROS 


\section{Prefácio}

Os sistemas em conformidade com as orientações do Current Research Information System (Sistema de Informação para Pesquisas Correntes CRIS) têm se apresentado como uma inovação no cenário mundial, que cada vez mais requer informações integradas e acrescidas de valor. É nesse contexto que se apresenta o DSPace-CRIS, uma ferramenta livre, de código aberto, que agrega funcionalidades voltadas à gestão da informação adequadas para construção dos chamados CRIS-locais, sistemas criados para a oferta de informações sobre pesquisas correntes e passadas, financiamento de projetos de pesquisa, caracterização e descrição das instituições de ensino e pesquisa, assim como de suas infraestruturas de pesquisa.

Entretanto, para que as instituições adotem essa nova tecnologia, requer-se conhecimento tecnológico sobre a ferramenta. Esse ponto pode apresentar certo desafio, pela escassa literatura em língua portuguesa e a pouca familiarização das equipes de informática com os sistemas científicos e tecnológicos.

Diante desse quadro, o Instituto Brasileiro de Informação em Ciência e Tecnologia (Ibict) convergiu esforços na produção deste guia. Com isso, procura contribuir com informações práticas e validadas pela equipe dessa instituição, com apoio da equipe mantenedora do software em nível internacional.

O Guia de Instalação do DSpace-CRIS descreve, de forma didática, um passo a passo de instalação, com explicações para cada etapa. Com isso, visa apoiar as equipes de informática das instituições que vierem a adotar este sistema, sobretudo no conhecimento inicial da ferramenta, com dicas nas questões mais cruciais, fruto dos problemas e soluções enfrentados no estudo do DSpace-CRIS pela equipe do Ibict.

Reforça-se, dessa maneira, o compromisso deste instituto com a comunidade brasileira, mediante a oferta de documentação técnica sobre uma solução informatizada, que apoie a disseminação de informações sobre pesquisa. Ressalta-se o caráter inovador da instituição, prospectando novas tendências mundiais.

Paulo César Egler 


\section{Apresentação}

Este guia visa orientar as instituições públicas e privadas do Brasil na instalação e na utilização da ferramenta livre DSpace-CRIS, versão 5.x, disponibilizada inicialmente pela Cineca e atualmente mantida pela 4Science. De maneira didática, são apresentadas ações necessárias para a instalação e configurações essenciais do software, desde a preparação do ambiente até o passo a passo para a instalação e customizações da aplicação.

O trabalho é resultado dos estudos vinculados ao projeto Current Reseach Information System Brasil (BRCRIS), desenvolvido no Brasil pelo Instituto Brasileiro de Informação em Ciência e Tecnologia (Ibict), instituição ligada ao Ministério da Ciência, Tecnologia, Inovações e Comunicações. O guia busca contribuir com a comunidade acadêmica ou científica na busca de soluções tecnológicas simples, práticas e fáceis, para implantar ou otimizar repositórios institucionais próprios.

Nos últimos anos, uma nova aplicação para repositórios denotada como DSpace-CRIS foi projetada com a função de expandir as funcionalidades do DSpace Padrão oferecendo informações sobre pessoas, projetos e organizações. Desta forma, oportuniza que a gestão, o manejo e o controle de todo o processo seja feito de modo bastante prático, facilitando atender, de maneira singular, as especificidades de cada instituição e de cada repositório em questão.

Considerando a escassa literatura técnica sobre o DSpace-CRIS em língua portuguesa, o guia apresenta-se como um documento valioso para que a comunidade acadêmica e científica possa ter maior conhecimento sobre o software e, consequentemente, para que haja maior expansão dos acervos digitais e uma participação mais efetiva de toda a sociedade no uso e acesso da população ao conhecimento. 


\section{0 que é o DSpace-CRIS}

O DSpace é um software livre destinado à criação de repositórios digitais. Foi desenvolvido pelo Massachusetts Institute of Technology (MIT), juntamente com a empresa Hewlett-Packard (HP), ambas norteamericanas. Destina-se, prioritariamente, a criar repositórios institucionais acadêmicos para disseminação de literatura científica de autores com vinculação a uma instituição, ofertando documentos geralmente já publicados em outros meios.

O Brasil tem sido um grande usuário dessa ferramenta. Desde 2005, a equipe técnica do Ibict apoia o DSpace de forma gratuita, para toda a comunidade acadêmica e científica do Brasil, dando suporte a usuários interessados em compartilhar documentação digital por meio da criação de repositórios institucionais acadêmicos.

O software também tem sido largamente utilizado pela esfera governamental, não como fonte primária, mas como instrumento de disseminação de documentos publicados previamente, iniciativa constatada pelos estudos de Brito, Macedo e Shintaku (2015).

Os Repositórios Digitais (RD) ou os Repositórios Institucionais (RI) atuam como sistemas digitais para preservação e disseminação de documentação de instituições - públicas ou privadas - ofertando acesso gratuito ao texto completo.

São, portanto, ferramentas oriundas do movimento mundial em direção ao "acesso aberto". Com isso, como relatam De Castro, Shaerer e Summann (2014), atendem as instituições que fornecem informações sobre as suas pesquisas, com todos os elementos envolvidos, sem passar pela necessidade de financiamento. Esses autores analisaram a questão entre RIs e a necessidade de gestão da informação de pesquisa - que é o principal objetivo do sistema CRIS - e constataram que as funcionalidades proporcionadas pelo CRIS decorrem do processo de evolução das ferramentas para criação das RIs.

O DSpace-CRIS é um projeto desenvolvido pela Cinecal para a Universidade de Hong Kong, que incorpora funcionalidades voltadas a projetos e pesquisadores. Recentemente (desde julho de 2016), os desenvolvedores originais do DSpace-CRIS bem como do DSpace mudaram

${ }^{1}$ http:// www.cineca.it 
para 4Science2, a qual continua a apoiar abertamente o projeto, fornecendo melhorias e customizações no repositório Github3 para ambos os repositórios.

O DSpace-CRIS expande as funcionalidades do DSpace padrão, agregando funcionalidades relacionadas a pessoas, projetos e organizações. Como mostra a Figura 1, sustentando-se nos seguintes pilares: Pessoas (pesquisadores, autores e outros), Outras Entidades (organizações e projetos) e Publicações (resultados de pesquisa como artigos, livros, relatórios e outros), a fim de ofertar informações consolidadas.

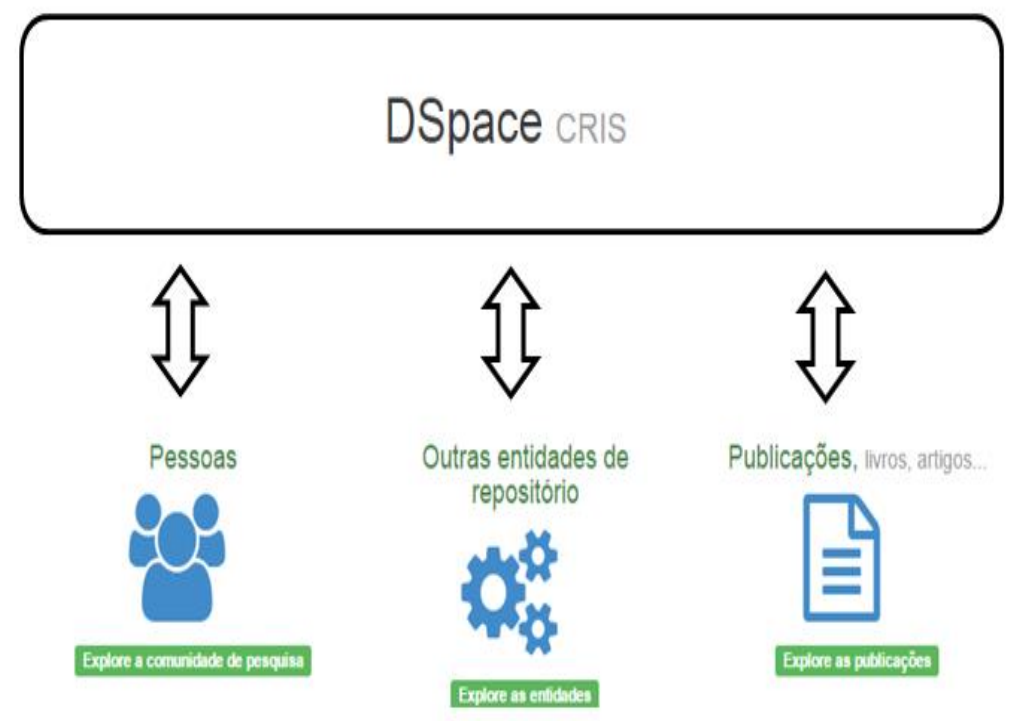

Figura 1 - Pilares do DSpace-CRIS

Palmer et al (2014) revelam que a adoção do DSpace-CRIS pela Universidade de Hong Kong, utilizando o padrão de metadados Common European Research Information Format (CERIF), não apenas aumentou a visibilidade das pesquisas da instituição, mas possibilitou o acesso facilitado a informações sobre colaboração entre os pesquisadores da instituição e a avaliação das pesquisas.

2Empresa italiana, com provedor de serviço registrado em:

http://www.dspace.org/service-providers

3 https://github.com/4Science/DSpace 


\section{Montar a Infraestrutura}

O DSpace-CRIS é um software livre, de código aberto, desenvolvido em Java Server Page, mas que agrega outras ferramentas, o que incrementa as suas funcionalidades. Isto faz com que, em sua instalação, sejam necessários todos os pré-requisitos para o seu pleno funcionamento.

A Figura 2 apresenta a estrutura de ferramentas necessárias para a instalação, manutenção e funcionamento do DSpace-CRIS. Todo o guia é compatível com sistemas operacionais ubuntu e debian (open sources). As ferramentas de apoio são indicadas pelos respectivos desenvolvedores originais do DSpace-CRIS e da DuraSpace mantenedora do DSpace padrão.

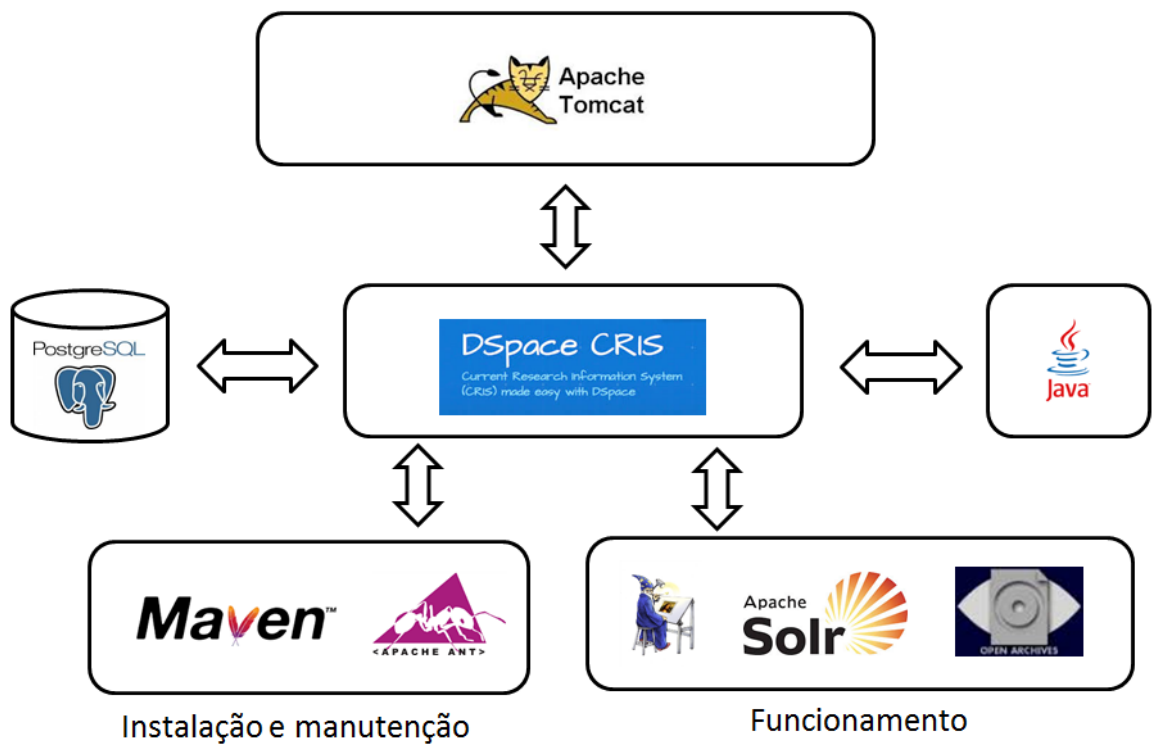

Figura 2 - Ferramentas necessárias

Os elementos necessários para a operacionalização do DSpace-CRIS são:

a) Infraestrutura básica:

- Sistema operacional open source, conforme a política do DSpace em relação a software livre; 
- Banco de dados para armazenamento dos registros;

- Servidor de Aplicação TomCat, como sugerido pela DuraSpace;

- Máquina Virtual Java (JVM) para execução dos programas compilados.

b) Apoio à instalação e manutenção:

- Apache-Maven para compilar o pacote contendo o fonte da aplicação;

- Apache-Ant para realizar a instalação do pacote compilado da aplicação;

c) Apoio às funcionalidades:

- ImageMagic para apresentar as miniaturas de capa dos documentados armazenados, principalmente com o formato PDF;

- SOLR, para implementar a ferramenta de busca;

- Protocolos OAI-PMH e OAI-ORE voltados à interoperabilidade.

A seguir, serão explicitados os requerimentos com relação à infraestrutura necessária para que o DSpace-CRIS possa ser instalado, bem como a pós-instalação, onde se configuram os módulos extras que são incorporados à versão "leve" do DSpace para versões Linux.

\subsection{Criar um usuário}

Para que a aplicação não fique espalhada por vários diretórios no servidor, com diversos tipos de permissões de acesso, recomenda-se a criação de um usuário e de um grupo de sistema que passe a ser o "dono" da aplicação (Owner).

Sugere-se a instalação com o nome de usuário e grupo usuariocris. Os comandos para criação do grupo usuariocris e usuário usuariocris devem ser executados como usuário root e são os seguintes:

$$
\text { addgroup - usuariocris }
$$

O comando acima cria um grupo para agrupar o usuário. 


\section{useradd $-\mathrm{m}$ usuariocris $-\mathrm{g}$ usuariocris}

Caso crie outro usuário, deve alterar em todos os comandos que se referirem a esse usuário.

\section{passwd usuariocris}

A senha pode ser qualquer uma, com o cuidado de mantê-la.

\subsection{Instalar o Banco de Dados}

O DSpace-CRIS necessita de uma base de dados para armazenamento dos registros, tal aplicação oferece compatibilidade com SGBDs Oracle e PostgreSQL.

Todavia, por ser um software livre, recomenda-se utilizar o PostgreSQL, alinhando as orientações governamentais ao software. Independentemente da escolha do SGBD, as melhores práticas de segurança aconselham a não manter a aplicação e o SGBD instalado na mesma máquina, além do que, separando-os, a aplicação tende a apresentar melhor performance de execução. A seguir daremos instruções para que o usuário possa escolher qual padrão deseja utilizar.

Logado como o usuário root, deve-se executar o seguinte comando para instalação do PostGreSQL:

\section{apt-get install postgresql}

Recomenda-se executar um apt-get-u update antes, para atualização da lista de instalação.

Após a instalação, acesse o arquivo: 
/etc/postgresql/x.x/main/postgresql.conf(x.x representa o número da versão Postegresql) e retire o \# (comentário) da linha abaixo:

\section{\#listen_addresses $=$ 'localhost'}

Com isso, o PostGreSQL pode ser acessado pelo próprio servidor (localhost) ou por um outro externo. Caso o banco esteja instalado em outro servidor, deve ser alterado para dar acesso apropriado, como segue:

\section{\#listen_addresses = 'IP_da_máquina'}

Posteriormente (ainda como root) acesse o arquivo /etc/postgresql/x.x/main/pg_hba.conf e adicione ao final do arquivo a linha.

a) caso esteja utilizando o banco no servidor local:

host all all 127.0.0.1 255.255.255.255 mat5

b) caso o banco esteja em um servidor externo:

host all all IP_da_máquina 255.255.255.255 md5

\subsection{Criar a Base de Dados}

Nesta etapa será criada a base de dados para a aplicação no banco de dados PostGreSQL, para armazenar os registros.

Nesse procedimento será criada apenas a base de dados, sem inserção de informação. 
Esta base de dados deve ter como proprietário um usuário de banco de dados, então, deve-se criar esse usuário primeiro e, posteriormente, a base de dados.

Para a criação do usuário de banco (logado como root), use os seguintes comandos:

\section{su postgres}

O comando acima troca para o usuário postgres.

$$
\text { createuser }-\mathrm{d}-\mathrm{A}-\mathrm{P} \text { usuariocris }
$$

O comando acima cria o usuário de banco de dados usuariocris, o qual será utilizado na aplicação.

$$
\text { exit }
$$

O comando acima volta para o usuário root. 
Para criar a base de dados, execute os seguintes comandos:

$$
\text { su usuariocris }
$$

O comando acima troca para o usuário usuariocris.

$$
\text { createdb -E UNICODE BDCris }
$$

O comando acima cria a base de dados BDcris, com formato de dados UTF-8 (unicode).

\subsection{Instalar a Java Virtual Machine (JVM)}

O Java Development Kit (JDK) promove a portabilidade das aplicações desenvolvidas em Java. Existem versões open source dessa ferramenta, como o Open-JDK, adotada neste guia.

Por uma questão de compatibilidade recomenda-se utilizar a versão 1.7 (a versão 1.8 costuma acarretar problemas). Execute o seguinte comando:

apt-get install openjdk-7-jdk openjdk-7-doc openjdk7-jre-lib

\section{NOTA}

Se ao utilizar o comando apt-get install, e algo der errado, poderá ser necessário reiniciar o processo. Isto é muito mais simples: apenas dê o comando apt-get purge remove nomedoprograma (-purge exclui todos os pacotes adicionais). 
Em seguida, confira se a versão corrente do JDK instalada está correta. Caso apareça outra versão, é provável que já havia uma JVM instalada. Para solucionar o problema, consulte o website Ubuntu Dicas ${ }^{4}$.

Para conferência da versão da JVM, execute os comandos abaixo:

$$
\text { java -version }
$$

e

javac -version

Os resultados de ambos deve apresentar a versão 1.7 do open-JDK.

${ }^{4}$ http:// www.ubuntudicas.com.br/2013/03/comando-para-escolherversoes-de-java-no-seu-ubuntu/ 


\section{Preparar o Ambiente e Compilar}

O DSpace-CRIS requer alguns procedimentos preparatórios para a sua instalação. Esta seção apresenta os procedimentos, aqui denominados preparação do ambiente e compilação. Com isso, espera-se descrever sucintamente todos os processos de instalação.

O DSpace-CRIS requer três diretórios para funcionamento, com as seguintes denominações:

Diretório Base: é o diretório em que a aplicação ficará hospedada, contendo arquivos de configuração, arquivos de log, etc da aplicação.

Diretório de Instalação: é o diretório em que é baixado o fonte, feita a configuração básica e disponibilizada a manutenção do sistema.

Diretório Web: é o diretório em que a aplicação web fica. 


\subsection{Criar o Diretório Base}

O Diretório Base geralmente fica no diretório raiz e, por isso, os comandos para a sua criação devem ser efetuados com o usuário root. Assim, logado como root, crie o diretório na raiz para acomodar a instalação do DSpace-CRIS com o seguinte comando:

mkdir /dspacecris-base

O nome do diretório a ser criado pode ser mudado, no entanto, mantenha o nome nas configurações.

Em seguida, modifique as permissões de acesso do diretório criado, mudando o usuário proprietário do diretório, com o seguinte comando:

chown usuariocris:usuariocris/dspacecris-base

\section{NOTA}

O passo acima é de suma importância, uma vez que é neste usuário que o DSpace-CRIS irá efetuar todos os procedimentos, requerendo permissões para alterar e criar arquivos nesse diretório.

\subsection{Criar o Diretório Fonte}

Denota-se diretório fonte o diretório utilizado na instalação e manutenção do DSpace-CRIS. Por padrão, tal diretório deve ser criado em /home/usuariocris, uma vez que esse usuário é o proprietário de toda a aplicação. Para baixar o código-fonte, certifique-se de estar logado com o usuário: usuariocris. O código-fonte está disponível no website Github $^{5}$.

${ }^{5}$ https://github.com/4Science/DSpace/tree/dspace-5_x_x-cris 
O diretório que armazenará o fonte da aplicação será referido nesse guia como DSpaceCRIS-fonte, constituído por um conjunto de arquivos de configuração, bem como os arquivos escritos na linguagem a qual a aplicação foi construída (Java Server Page), e pode ser acessado pelo caminho:

\section{cd/home/usuariocris/DSpaceCRIS-fonte}

\subsection{Configurar o DSPace-CRIS para instalação}

Para que se possa iniciar a compilação, é necessário acessar o arquivo de configuração que se encontra no /home/usuariocris/DSpaceCRIS-fonte /build.properties. Este arquivo contém os parâmetros necessários para adequar a instalação, bem como configurações mais específicas que não serão detalhadas aqui. Essas são as configurações iniciais e necessárias para a instalação.

Ajuste as configurações conforme o Quadro 1. No processo de compilação, as informações colocadas no arquivo build.properties são copiadas para os arquivos de configurações destino. Caso deixe de informar alguma variável, pode causar problemas na instalação. Essas informações podem ser alteradas posteriormente. 


\section{VARIÁVEIS}

dspace.install.dir=/dspacecris-base

dspace.hostname $=$ localhost

dspace.baseUrl $=$ http: $/ /$ localhost: 8080

dspace. name $=$ DspaceCRIS

dspace.ui $=$ jspui

db.url=jdbc:postgresql : //localhost: 5432/BDcris db.username=usuariocris

$\mathrm{db} \cdot$ password $=\mathrm{xxxxx \textrm {x }}$

mail.server $=$ smtp. example.com

mail.server.username=

mail.server.password=

mail.from.address=email.edu.com

mail. feedback. recipient = email.edu.com

mail.admin = email.edu.com

mail.alert.recipient=email. edu.com

mail.registration. notify=email.edu.com

\section{CONFIGURAÇÕES}

Diretório para instalação criado no passo 3.1

Nome do servidor na rede

Endereço do servidor na rede ou quando configurado o DNS a URL especificada

Nome do Repositório

A interface que o DSpace-CRIS utiliza

Nome do banco, usuário e senha criados no passo 2.3

Servidor de e-mail para o DSpace-CRIS enviar mensagens automáticas;

Usuário e senha do servidor de e-mail

Endereço de e-mail do responsável por receber os e-mails disparados pelo DSpace-CRIS

E-mail para receber as mensagens de feedback dos usuários;

E-mail do administrador do repositório

E-mail que receberá os erros de sistema

E-mail que receberá a notificação de registro de um novo usuário

Quadro 1-Varáveis com as configurações correspondentes 
Como padrão, utiliza-se a porta 8080 para acessar a aplicação externa ou internamente na rede pelos browsers. Todavia é importante verificar se a porta não se encontra em uso. Além disso, é possível configurar outra porta.

Para verificar se a porta está em uso, certifique-se de estar como root, e execute o seguinte comando:

\section{cnetstat -tlpn | 8080}

O comando acima verifica se a porta 8080 está sendo utilizada. Se não listar nada no terminal, a porta está livre. Caso contrário, será listado o programa que está fazendo uso da mesma.

\subsection{Compilar o DSpaceCRIS-fonte}

O Apache-Maven é a ferramenta que realiza a construção de toda a árvore de pacotes que serão necessários para instalação e compilação do código fonte referente ao DSpace-CRIS. Para a instalação do ApacheMaven, logado como usuariocris, deve-se baixar o seu pacote binário no website do Apache-Maven ${ }^{6}$ e descompactá-lo em /home/usuariocris/ por meio do comando (substitua x.x.x pelo número da versão baixada):

\section{tar -vzxf apache-maven-x.x.x-bin.tar.gz}

Em alguns casos, as instituições requerem o uso de servidor de Proxy para intermediar o acesso de um usuário na rede local e a rede externa (internet de modo geral). Assim, caso o servidor encarregado de hospedar a aplicação utilize autenticação via servidor proxy, será necessário criar um diretório de nome .m2 dentro de /home/usuariocris/, e dentro do diretório .m2 criar um documento de nome settings. xml com o seguinte conteúdo.

${ }^{6}$ http:// maven.apache.org/ 


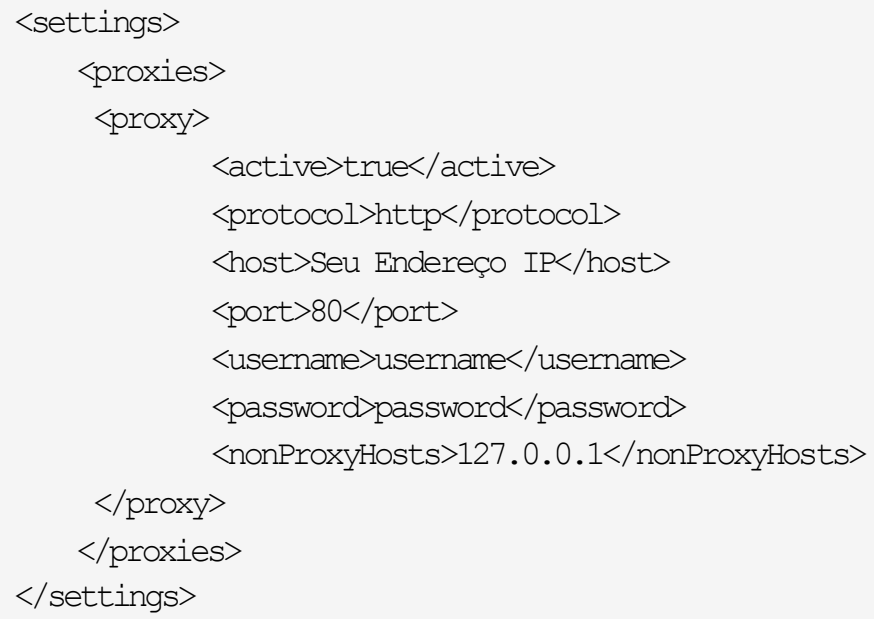

$\mathrm{O}$ username e password são o login e senha de autenticação no proxy.

\subsection{Instalar o ImageMagick}

Nativamente, o DSpace-CRIS não cria as miniaturas das capas dos documentos PDFs depositados. Deste modo, tem-se a necessidade de instalar uma ferramenta denotada como ImageMagick para este fim.

Execute o seguinte comando via terminal logado como root:

apt-get install imagemagick

Finalmente, para compilar o fonte do DSpace-CRIS, assegure que esteja logado como usuário usuariocris, navegue pelo terminal até /home/usuariocris/DSpaceCRIS-fonte e execute o comando abaixo: 
A instalação se desenvolve por no máximo 15 minutos e o sistema deverá retornar na tela a confirmação de sucesso denotada como BUILD SUCCESSFUL. 


\section{Instalar o DSpace-Cris}

O processo de instalação deve ser realizado com login de usuário usuariocris. A ferramenta que executa a tarefa de instalação do DSpace-CRIS é o Apache-Ant, disponível (pacote binário) em http://ant.apache.org/. Por questão de organização, é aconselhável descompactá-lo dentro de /home/usuariocris.

A instalação é executada dentro de:

$\mathrm{cd} /$ home/usuariocris/DSpaceCRIS-

fonte/dspace/target/dspace-installer

Por meio do comando:

/home/usuariocris/apache-ant-x.x.x/bin/ant fresh_install

Após, no máximo 5 minutos, o sistema deve responder com um log na tela com a mensagem BUILD SUCCESSFUL.

Caso ocorra algum problema e uma mensagem de erro seja apresentada, pode-se refazer o processo, corrigindo o erro. Para tanto devese limpar a compilação existente com o seguinte comando:

\section{/home/usuariocris/apache-ant-x.x.x/bin/ant clean/}

$O$ processo de instalação pode ser refeito $n$ vezes. Todavia, certifique-se de sempre utilizar o ant clean para limpar os erros da compilação anterior. 


\section{Pós-instalação do DSpace-CRIS}

Após a implantação do DSpace-CRIS, é necessário que se crie uma senha de administrador. Logado como usuariocris, navegue pelo terminal até /dspacecris-base/bin/ e execute o seguinte comando:

\section{./dspace create-administrator}

O administrador é o responsável pelo controle de segurança bem como pelas operações rotineiras que o sistema necessita.

\section{NOTA}

O DSpace-CRIS orienta a criação vários administradores por questões gerenciais. 


\section{Configurações}

Por padrão, a configuração de dados DSpace-CRIS é vazia. Todavia, é possível utilizar um arquivo do Excel para configurar o sistema com as definições padrões que se fazem necessárias. Para isto, logado como usuariocris, navegue até /dspacecris-base/bin/ e execute duas vezes o comando:

./dspace load-cris-configuration -f / dspacecrisbase/etc/configuration-tool-demo.xls 


\section{Iniciar o DSpace-CRIS}

Com o sistema instalado, o primeiro passo é colocar a aplicação disponível aos usuários na Web.

Deste modo, necessita-se de um servidor para acomodar o DSpace-CRIS. Recomenda-se utilizar o tomcat para tal tarefa.

O tomcat é um servidor Web que torna disponível o acesso de aplicações via rede (seja Intranet ou Internet).

Logado como usuariocris, baixe o pacote binário do disponível no website do tomcat $^{7}$ e descompacte-o dentro de /home/usuariocris/. É necessário que se faça uma mudança na codificação de apresentação dos arquivos, acessando o arquivo (x.X.X representa a versão do tomcat):

nano/home/usuariocris/apache-tomcat-x.x.x/conf/server.xml

E em seguida, inserindo o termo URIEncoding="UTF-8" no seguinte conector:

$<$ Connector port="8080" protocol="HTTP/1.1"

connectionTimeout="20000"redirectPort="8443" URIEncoding="UTF-8" >

A ativação do servidor tomcat com a aplicação do DSpace-CRIS se dá por meio de cópias dos módulos apps do diretório de instalação para o tomcat, como segue:

${ }^{7}$ http://tomcat.apache.org/ 
qp -R /dspacecris-base/webapps/jspui//home/usuariocris/apachetomcat-X.X.X/webapps/

cp -R /dspacecris-base/webapps/solr/ /home/usuariocris/apachetomcat-x.x.x/webapps/

cp -R /dspacecris-base/webapps/oai//home/usuariocris/apachetomcat-x.x.x/webapps/

Acima, foram copiados unicamente os módulos que compõem o funcionamento básico do DSpace-CRIS. Caso o usuário tenha interesse em utilizar todos os módulos do DSpace-CRIS, copie-os dos módulos encontrados em /dspacecris-base/webapps/ com o seguinte comando:

cp -R /dspacecris-base/webapps/*/home/usuariocris/apache-tomcat$\mathrm{X} . \mathrm{x} . \mathrm{x} /$ webapps/

Em última instância, o tomcat necessita de uma configuração. Baixe o tomcat (caso esteja ativo):

/home/usuariocris/apache-tomcat-x.x.x/bin/shutdown.sh

Crie um arquivo de nome setenv.sh no diretório:

/home/usuariocris/apache-tomcat-x.x.x/bin/ 
Insira a seguinte linha:

CATALINA OPTS="-Dorg $\cdot$ apache.el $\cdot$ parser.SKIP

IDENTIFIER CHECK=true -XX:PermSize=256m -Xms256m -Xmx2048m"

\section{NOTA}

É possível mudar os parâmetros de memória -Xms256m $\mathrm{Xmx} 2048 \mathrm{~m}$ de acordo com as especificações de hardware utilizado.

Salve o arquivo, e suba novamente o tomcat (/home/usuariocris/apache-tomcat-x.x.x/bin/start.sh).

\section{NOTA}

Para um desempenho recomenda-se executar o SOLR em um tomcat separado. Você pode utilizar o mesmo pacote tomcat instalado para o DSpace com um catalina_home diferente. Porém, ao escolher essa opção, tenha certeza de atribuir portas diferentes (e. g. 8081 , etc) aos conectores do tomcat e altere, também, os parâmetros no discovery.cfg ( /dspacecrisbase/config/ ) 


\section{Customizações}

A seguir será compartilhado o conhecimento adquirido pela equipe destinada à pesquisa do DSpace-CRIS. Deve-se atentar para que tudo que seja realizado na aplicação seja feito com usuariocris, salvo existir alguma ressalva.

\subsection{Adicionar o Idioma Português do Brasil}

Por uma questão de padronização, o DSpace-CRIS utiliza o idioma inglês.

Como contribuição, o Ibict está disponibilizando uma tradução em português na Wiki $\mathrm{Ibict}^{8}$. Os passos necessários para que o DSpace-CRIS reconheça o arquivo de tradução são os seguintes:

a) Abra o arquivo /dspacecris-base/config/dspace.cfg;

b) Localize default.language $=$ en e substitua por default.language $=$ pt_BR;

c) Localize default. locale $=$ en e substitua por default. locale $=$ pt_BR;

d) Localize webui.supported. locales $=$ en e substitua por

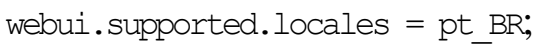

e) Copie o arquivo de tradução para:

/home/usuariocris/apache-tomcat-x.x.x/webapps/jspui/WEB-INE/classes/

Atente para as permissões de usuário, ou seja, observe se o arquivo adicionado é de posse do usuariocris utilizado para executar a aplicação. A mudança de posse, caso seja necessário, é feita utilizando o seguinte comando chwon usuariocris:usuariocris "nome do arquivo de tradução".

Por fim, pare o tomcat e reinicie-o novamente.

${ }^{8}$ wiki.ibict.br/index.php/DSpace-CRIS 


\subsection{Busca Interna nos Arquivos Armazenados}

O DSpace-CRIS pode ser configurado para buscar trechos de texto interno aos arquivos mantidos no repositório. A seguir serão descritos os passos necessários para que se possa configurar tal serviço.

a) Certifique-se de estar logado com o usuariocris.

b) Abra o arquivo /dspacecris-base/config/dspace.cfg

- Defina search.maxfieldlength $=-1$

- Execute o seguinte comando /dspacecris-base/bin/dspace filter-media para que a aplicação reconheça o serviço.

- Por fim, pare o tomcat (/home/usuariocris/apache-tomcat-x.x.x/bin/shutdown.sh) e reinicie-o novamente (/home/usuariocris/apache-tomcat-x.x.x/bin/start.sh).

\subsection{Configurar Serviço de E-mails}

A configuração será feita levando em consideração o servidor de e-mail do Gmail. Todavia é possível utilizar um servidor de e-mail corporativo.

Logado como usuariocris, configure o serviço de e-mail do repositório como segue.

\subsubsection{Criar conta de e-mail}

Crie uma conta no Gmail para controlar o serviço de e-mail do repositório. Aconselha-se a registrar apenas uma conta nos demais parâmetros citados abaixo. 


\section{Crie sua Conta do Google}
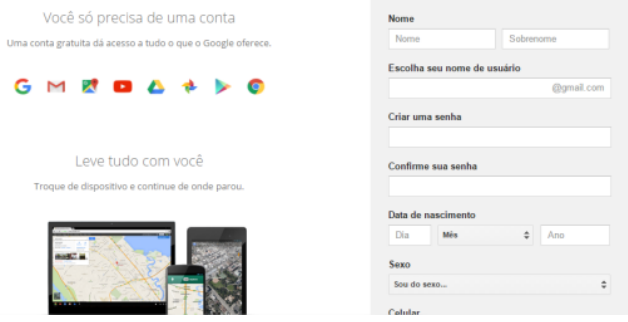

Figura 3 - Página inicial para criação da conta Gmail

\subsubsection{Definir parâmetros}

Abra o arquivo /dspacecris-base/config/dspace.cfg. Em seguida, defina os seguintes parâmetros:

\begin{tabular}{|c|c|c|}
\hline COMENTÁRIO & PARÂMETRO & VALOR \\
\hline $\begin{array}{l}\text { Inserir um servidor de smtp } \\
\text { válido }\end{array}$ & mail.server $=$ & smtp.Gmail.com \\
\hline Usuário & $\begin{array}{l}\text { mail.server.username } \\
=\end{array}$ & user.Gmail.com \\
\hline Senha & $\begin{array}{l}\text { mail.server.password } \\
=\end{array}$ & $x x x x$ \\
\hline $\begin{array}{l}\text { Porta para acessar o } \\
\text { servidor smtp }\end{array}$ & mail.server.port $=$ & 465 \\
\hline $\begin{array}{l}\text { Informar o endereço para e- } \\
\text { mail. }\end{array}$ & mail.from.address = & user.Gmail.com \\
\hline $\begin{array}{l}\text { Limitado a um único } \\
\text { recipiente }\end{array}$ & feedback. recipient = & user.Gmail.com \\
\hline Administração geral do site & mail.admin $=$ & user.Gmail.com \\
\hline Para erros e alertas & alert.recipient $=$ & user.Gmail.com \\
\hline $\begin{array}{l}\text { Notificação de novos } \\
\text { usuários cadastrados }\end{array}$ & registration.notify $=$ & user.Gmail.com \\
\hline
\end{tabular}




\section{Quadro 2-Parâmetros}

\subsubsection{Configurar conta de e-mail}

Configure a conta no Gmail para que aceite as requisições clicando em minha conta.

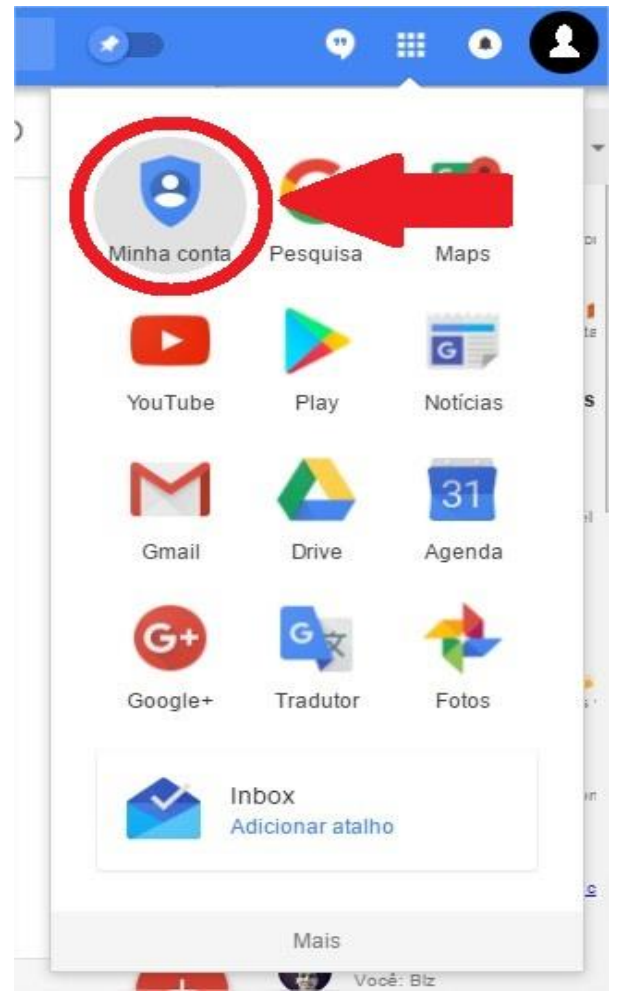

Figura 4 - Janela GoogleApps 
Em seguida, clique em Verificação de segurança.

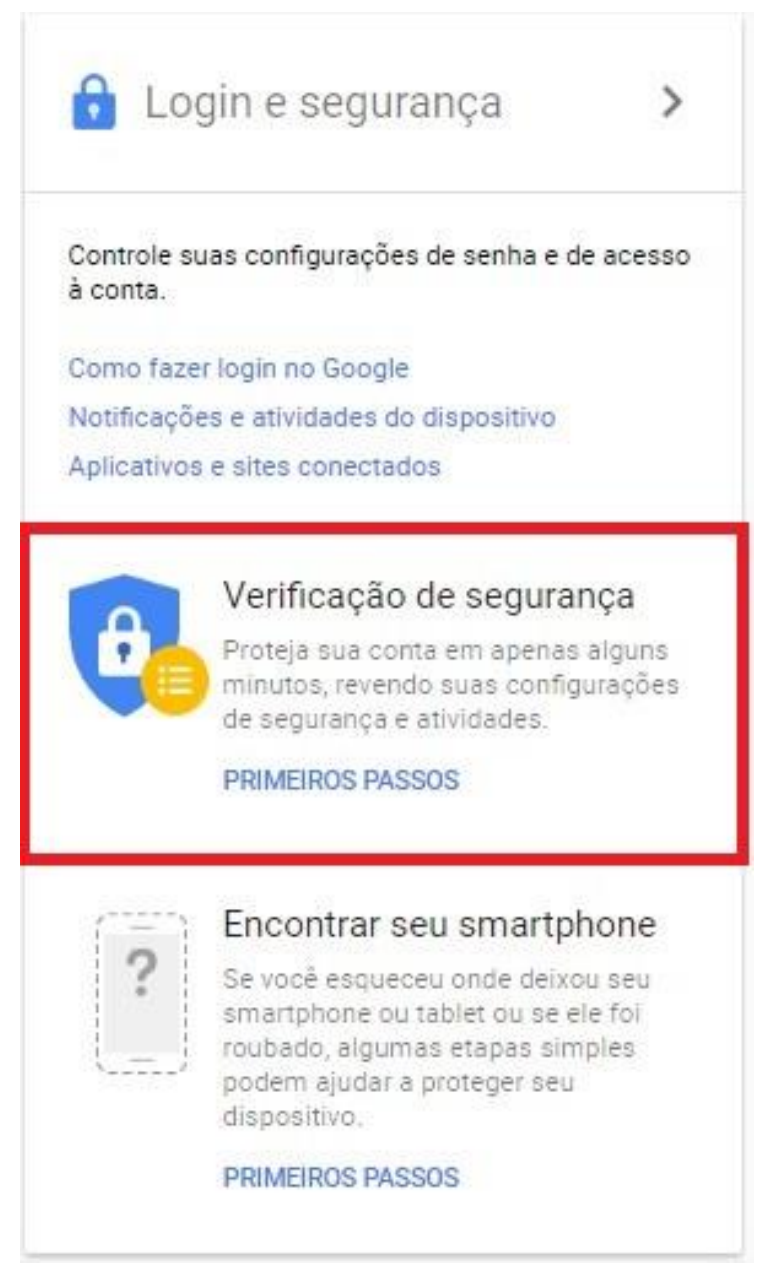

Figura 5 - Janela 'Minha Conta'

Por fim, clique para ativar o serviço. Isso torna a conta menos segura, todavia sem ativar o acesso para aplicativos menos seguros, o usuariocris não poderá utilizar o servidor do Gmail para validação. 


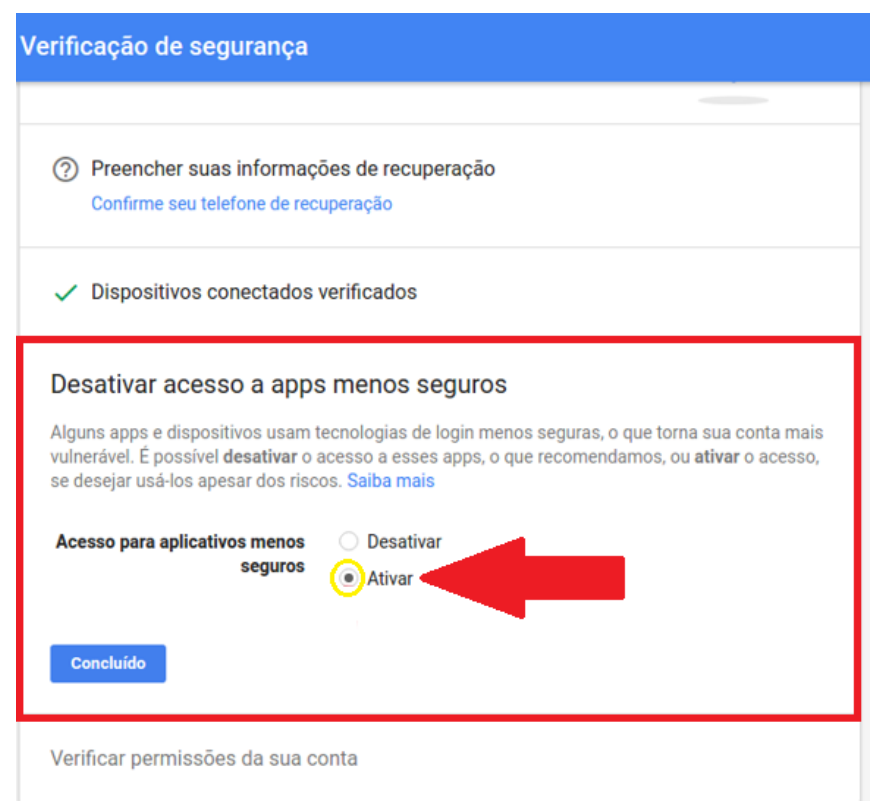

Figura 6 - Janela Verificação de segurança

Por fim, pare o tomcat utilizando o comando:

/home/usuariocris/apache-tomcat-x.x.x/bin/shutdown.sh

Em seguida reinicie-o, usando a linha de comando:

/home/usuariocris/apache-tomcat-x.x.x/bin/start.sh

Desta forma, o serviço estará apto ao uso.

Para personalizar as mensagens a serem enviadas para os usuários, navegue até /usuariocris-base/config/emails/. Neste diretório existem diversos arquivos relacionados ao serviço de e-mail. $\mathrm{O}$ 
administrador do repositório pode configurar os arquivos à sua maneira, preenchendo os argumentos Subject e Comments.

\section{Considerações Finais}

A necessidade de expansão dos serviços ofertados pelos Repositórios Digitais apresenta desafios e oportunidades, na medida em que requer novos desenvolvimentos. Por esse motivo, o DSpace-CRIS se apresenta como uma opção para as instituições que desejam um repositório com maiores funcionalidades, apoiado por instituição consolidada.

Entretanto, uma das dificuldades na adoção dessa tecnologia ainda reside no pouco conhecimento por parte da comunidade de informática sobre essa ferramenta, além da escassa literatura técnica existente em português. Assim, esse guia fornece informações validadas para as equipes de informática que desejam utilizar o software, sem, no entanto, serem extensivas ou definitivas. Procura ofertar a informação inicial validada para instalação.

Assim, contribui com a comunidade de informática pouco acostumada com sistemas acadêmicos e de gestão da informação científica, apoiada na missão do Ibict em promover tecnologias voltadas à promoção da produção, socialização e integração do conhecimento científico e tecnológico. Esperase, posteriormente, contribuições da comunidade para melhoria e/ou novos materiais técnicos. 
Referências

CASTRO, Pablo de; SHEARER, Kathleen; SUMMANN, Friedrich. The Gradual Merging of Repository and CRIS Solutions to Meet Institutional Research Information Management Requirements. Procedia Computer Science, [s.1.], v. 33, p.39-46, 2014. Elsevier BV. http://dx.doi.org/10.1016/j.procs.2014.06.007. Disponível em: < http://www.sciencedirect.com/science/article/pii/S1877050914007972 >. Acesso em: 09 ago. 2016.

MACÊDO, Diego José; SHINTAKU, Milton; BRITO, Ronnie Fagundes de. Dublin Core usage for describing documents in Brazilian government digital libraries. In: DCMI International Conference on Dublin Core and Metadata Applications, 2015, São Paulo. Proceedings... São Paulo: DCMI, 2015. p. 129 - 135. Disponível em: < http://dcpapers.dublincore.org/pubs/article/view/3768/1958 >. Acesso em: 06 ago. 2016.

PALMER, David T. et al. DSpace-CRIS@HKU: Achieving Visibility with a CERIF Compliant Open Source System. Procedia Computer Science, [s.1.], v. 33, p.118-123, 2014. Elsevier BV. http://dx.doi.org/10.1016/j.procs.2014.06.019. Disponível em: < http://www.sciencedirect.com/science/article/pii/S1877050914008096 >. Acesso em: 09 ago. 2016.

DURASPACE. DSpace-CRIS Home. In: Wiki Duraspace. Disponível em: $<$ https://wiki.duraspace.org/display/DSPACECRIS/DSpace-CRIS+Home $>$ Acesso em 20 jul. 2016. 


\section{Sobre os Autores}

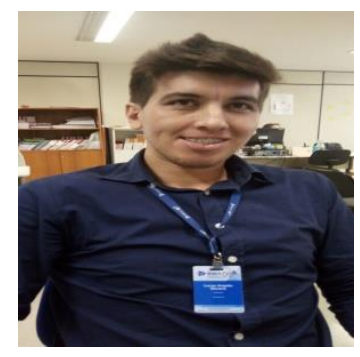

\section{Lucas Angelo da Silveira}

orcid.org/0000-0002-8107-9659

Doutorando em Informática pela Universidade de Brasília (UnB), atua no apoio ao DSpace e DSpace Cris a instituições não acadêmicas no lbict.

\section{Milton Shintaku}

orcid.org/0000-0002-6476-4953

Doutor em Ciência da Informação pela Universidade de Brasília (UnB).
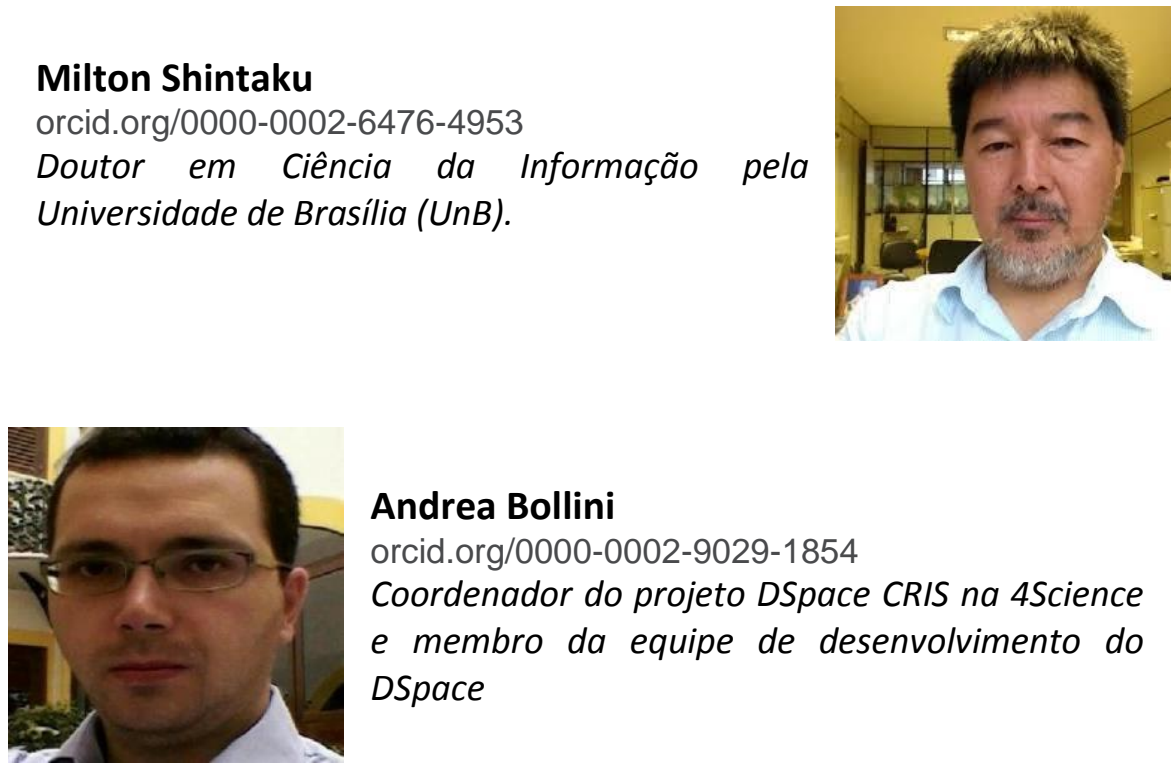

\section{Andrea Bollini}

orcid.org/0000-0002-9029-1854

Coordenador do projeto DSpace CRIS na 4Science

e membro da equipe de desenvolvimento do DSpace 


\section{Sobre o lbict}

O Ibict é uma unidade de pesquisa vinculada ao Ministério da Ciência, Tecnologia, Inovações e Comunicações, voltada à promoção da competência, ao desenvolvimento de recursos e à infraestrutura de informação em ciência e tecnologia para a produção, socialização e integração do conhecimento científico e tecnológico.

Criado em 1954, inicialmente como Instituto Brasileiro de Bibliografia e Documentação (IBBD), o Ibict efetua, dentre tantas iniciativas, ações de transferência de tecnologias para instituições públicas ou privadas do Brasil.

$\mathrm{O}$ apoio às ferramentas mantidas pelo Public Knowledege Project (PKP), por exemplo, tem influência significativa na criação de portais e revistas científicas de acesso aberto online em todo o país.

Esta ação é mantida, em sua grande parte, na Coordenação Geral de Tecnologia da Informação (CGTI), uma das três coordenações gerais do instituto, responsável pelas ações relacionadas à Tecnologia da Informação e Comunicação.

\section{Sobre a CGPD}

Coordenação Geral de Desenvolvimento e Pesquisa de Novos Produtos (CGPD) forma, juntamente com a Coordenação Geral de Tecnologia da Informação e com a Coordenação Geral de Pesquisa e Manutenção de Produtos Consolidados, a tríade de sustentação das ações do Ibict.

Cabe a CGPD desbravar os novos desafios do atual cenário, expandindo as fronteiras do Ibict, no que tange ao tratamento da informação. Assim, o projeto de ecossistema basileiro voltado a tratar das informações sobre as pesquisas correntes e passadas (BrCRIS) é de responsabilidade da CGPD, em cooperação estreita com as outas coordenações gerais.

Com isso, revela o vanguardismo da CGPD, na medida em que apoia o Ibict nas ações inovadoras. Oferta a comunidade brasileira novos produtos, resultantes de extensa pesquisa, contribuindo com a missão do Ibict. 


\section{Sobre a CGTI}

A Coordenação Geral de Tecnologia da Informação (CGTI) forma, juntamente com a Coordenação Geral de Pesquisa e Desenvolvimento de Novos Produtos e com a Coordenação Geral de Pesquisa e Manutenção de Produtos Consolidados, a tríade de sustentação das ações do Ibict.

A CGTI tem o objetivo de apoiar as atividades do instituto em relação às TICs, ofertando serviços de infraestrutura de informática e de uso de ferramentas. Atua nas diversas iniciativas apoiadas pelo Ibict, em ações de suporte, prospecção e transferência de tecnologias, mantendo canais abertos com as demais coordenações e com a comunidade externa.

É composta por duas coordenações e duas divisões, dentre as quais está a coordenação de Articulação, Geração e Aplicação de Tecnologia (COAT). Prioritariamente, cabe à COAT realizar ações voltadas à disseminação e à transferência de tecnologias, assim como desenvolver pesquisa aplicada.

\section{Sobre a COAT}

A COAT é a coordenação responsável por ofertar apoio às várias tecnologias desenvolvidas ou apoiadas pelo Ibict. Faz prospecção, absorção, disseminação e repasse de tecnologias que apoiem as atividades da instituição ou da comunidade científica brasileira. Para tanto, tem mantido iniciativas que atendam seus usuários de forma mais ampla, como publicações e sites.

Uma iniciativa importante da COAT é a Wiki do Ibict (http://wiki.ibict.br/index.php/P\%C3\%A1gina_principal), uma iniciativa colaborativa que visa ofertar orientações aos usuários das tecnologias apoiadas pelo Ibict.

A COAT promove ainda o "forum ibict" (http://forum.ibict.br/), um canal de interação entre os usuários externos e os técnicos da COAT. Neste canal, as dúvidas ou problemas da área, com as devidas soluções, ficam registradas, a fim de a apoiar a todos os usuários, consolidando uma vasta base de dados.

A COAT desenvolve ainda publicações, resultantes de estudos e pesquisas, para a disseminação de conhecimento sobre ferramentas livres. 
Com isso, apoia a missão da instituição, fornecendo informações validadas sobre soluções tecnológicas para toda a sociedade brasileira.

\section{Outras publicações}

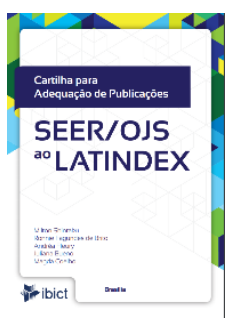

SHINTAKU, Milton et al. Cartilha para Adequação de Publicações SEER/OJS ao Latindex. Brasília: Ibict, 2014. 44 p.

SHINTAKU, Milton; BRITO, Ronnie F. de; FLEURY, Andrea. Cartilha SOAC/OCS para gerentes gerais. Brasília: Ibict, 2014. 64 p.
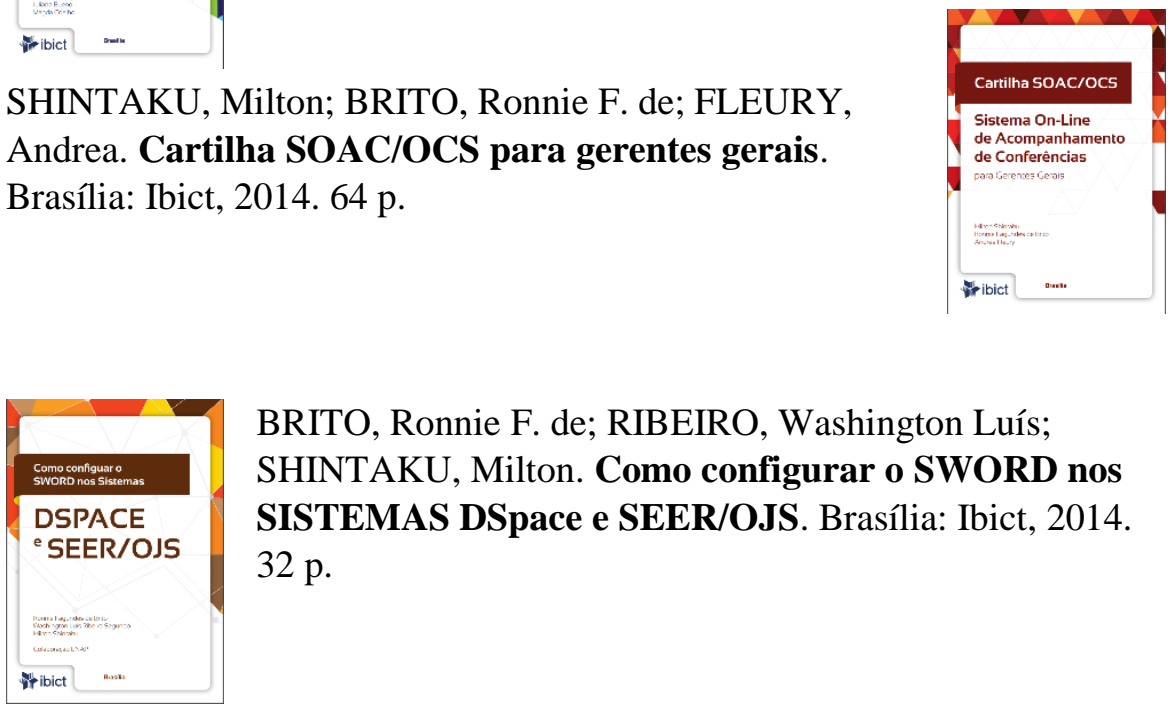

BRITO, Ronnie F. de; RIBEIRO, Washington Luís; SHINTAKU, Milton. Como configurar o SWORD nos SISTEMAS DSpace e SEER/OJS. Brasília: Ibict, 2014. $32 \mathrm{p}$.

BRITO, Ronnie F. de et al. Guia do Usuário do Digital Object Identifier. Brasília: Ibict, 2014. 57 p.

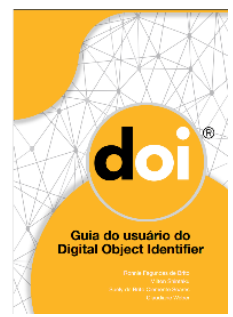


43 
A criação do Guia do DSpace-CRIS, pelo Instituto Brasileiro de Informação em Ciência e Tecnologia, reforça, mais uma vez,

o compromisso da instituição com o repasse permanente de conhecimento científico e tecnológico para a sociedade brasileira.

Otrabalho é fruto do esforço da equipe técnica do Ibict em oferecer alternativas científicas e tecnológicas voltadas a atender necessidades de instituições públicas e privadas em disponibilizar sistemas digitais que viabilizem a criação de repositórios institucionais e a consequente disseminação do conhecimento em acesso aberto. A ferramenta DSpace-CRIS apresenta-se como uma nova perspectiva de solução tecnológica para a oferta, pelas instituições, e para o acesso, pela sociedade, das informações científicas consolidadas em bancos de dados. O Guia do DSpace-CRIS, iniciativa que integra o Projeto do Ecossistema BRCRIS - matriz e berço do DSpace-CRIS significa, em síntese, mais uma contribuição do lbict para a eficiência da gestão da ciência brasileira.

Arthur Fernando Costa Coordenação Geral de Pesquisa e Desenvolvimento de Novos Produtos - IBICT

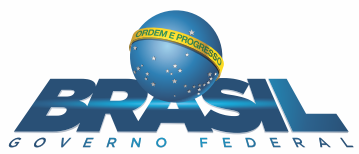

